Session 2024

\title{
Optical Design Tools for Photonics Engineering Education
}

\author{
Alexander N. Cartwright, Pratibha Gopalam, \\ Vamsy P. Chodavarapu and Bill Kirkey \\ Department of Electrical Engineering, \\ University at Buffalo, State University of New York, \\ Buffalo, NY 14260, USA.
}

\begin{abstract}
Java $^{\text {TM }}$ Educational Applets have demonstrated that they are powerful tools for the development of web-based educational materials and design tools because these resources can be accessed by anyone who has access to the World Wide Web (WWW) and they present the matter in dynamic visual appealing manner. In this paper, we will review a comprehensive set of photonics applets, an accompanying website and a necessary framework dealing with the development of optical design tools which are available for online teaching, experimentation and research. To date, the design tools developed cover ray tracing, Gaussian beams, polarization, gain medium and gain dynamics, modulators and optical cavities. In addition, the resources include a number of demonstration applets such as photon lifetime, wave interference, Snell's law and others. The main advantages of embedding design into optics courses, by using simulation software, are that the underlying and fundamental principles are made easy to understand by creating a platform on which students can experiment with the various facts and design new systems which may otherwise require a large and an expensive setup of laboratory equipment and at the same time making the theoretical courses more interesting to learn. Moreover, we are simultaneously attempting to develop tools that researchers can use to readily simulate complicated systems which may otherwise require hours of work.
\end{abstract}

\section{Introduction}

The photonics industry is growing at a very high rate in response to an increase in the market demand for new photonics equipment like fiber optic communications systems, optical scanners, displays and laser printers leading to a demand for skilled workers with hands-on experience and the ability to design and develop new systems.

"Proceedings of the 2002 American Society for Engineering Education Annual Conference \& Exposition Copyright (C2002, American Society for Engineering Education” 
In a paper previously published by us [1], we have described how to integrate the use of design in laser and photonics courses. We have implemented that strategy and in doing so, created a new virtual learning environment that has impact on three courses at the University at Buffalo, The State University of New York, which are taught for undergraduates (Lasers and Photonics, EE 492 and Consumer Optoelectronics EE494) and graduates (Optical Communications, EE 566 and Consumer Optoelectronics, EE594) respectively. In these courses the main emphasis is on the design of various optical systems.

The inclusion of design tools and multimedia technologies in these courses created an appealing virtual environment where the students can experiment with what they learn in lectures and understand better the fundamental principles underlying various photonic devices. Moreover, the development of hands-on experimental apparatuses for the study of fabrication, materials properties and optical characteristics are typically cost prohibitive. Simulations, on the other hand, can be very cost-effective to implement and consume less time and effort as compared to setting up a real laboratory.

However, as correctly pointed out by "Wallace and Mutooni" [2], merely presenting the material using World Wide Web based multimedia technologies does not guarantee that students will use it effectively. Therefore, in addition to developing the online materials, we have taken utmost care in seeing that all students can benefit from these resources by modifying the three photonics courses to address many of the guidelines pointed out by the 'Criteria for Accrediting Engineering Programs" [3] set by the Accreditation Board for Engineering and Technology (ABET).

It is a daunting task to develop sufficient simulations to cover all areas of photonics. The area of photonics is vast and overlaps with many diverse areas such as Semiconductor Physics, Opto-Electronics, MOEMS, Bio-Photonics, Polymeric materials, etc. Therefore, it is not possible for a single group to create a comprehensive set of design tools that can be used for all the areas. Moreover, as we have pointed out in the past, it is essential that the design of the functionality of a design applet be done by a subject matter expert [1]. Therefore, we have developed a set of applets that explain certain fundamental principles. All the applets are based on object oriented technologies and grouped into a single framework which can easily be disseminated to other faculty members [4]. In the near future, all the developed Java applets and the web-based courseware materials will be incorporated into a system-level top-down approach wherein the user of our courseware enters at a high level view of an everyday real-world photonic device and then selects components of that product that he/she wants to investigate. The user then interacts with specific java applets that describe the details of the selected component operation and design [5].

"Proceedings of the 2002 American Society for Engineering Education Annual Conference \& Exposition Copyright (C2002, American Society for Engineering Education” 


\section{Implementation of the New Learning Environment}

As stated earlier the New Learning Environment was developed by modifying three courses at the University at Buffalo which are conventionally taught exclusively as theory based lecture style courses elsewhere. The undergraduate course taught for seniors "Lasers and Photonics (EE 492)" expects the student to design a laser resonator based on a given gain medium's specifications as their final course project. During the course, the students are given assignments to design simple optical systems such as a multi- and single lens systems and optical fiber systems by using the design tools provided in the course web-site [6]. A few example questions are given below in Figure 1 and an example final solution for Problem 1 is shown in Figure 2.

"Use the Form Based Optical System Design v2.0 applet located at http://www.eeeng.buffalo.edu/ anc/photonics/photonics/OpticalDesignVer2/dynamicForm.html to do the following problems. Submit a printout of the completed form along with a hand sketch of the optical system."

\section{Problem 1}

Construct and demonstrate a beam compressor which reduces the spread of an incident plane wave by a factor of 3 .

\section{Problem 2}

Construct the optical system of problem 1.4-1 (choose an appropriate value for $\mathrm{f}$ ). Show light from both a plane wave and a point source propagating through the system.

\section{Problem 3}

Reconstruct your beam compressor from problem 1, but this time also add a dielectric with $n=1.5$ somewhere in between the two lenses. Notice that the output from the plane wave input is no longer a plane wave. Try to adjust the position of the output lens to obtain a plane wave output. Does this work? Explain.

\section{Problem 4}

A. Construct a cavity which consists only of two mirrors, each with a radius of curvature of -10 and a height of 30 . The mirrors should be separated by 5 and should face each other. Now add a plane wave originating outside the cavity. What do you notice about the behavior of the rays inside the cavity?

B. Repeat part A using a point source instead of a plane wave.

C. Repeat part A using a Gaussian beam instead of a plane wave.

For assistance you could also use the other applets located at http://wwwee.eng.buffalo.edu/ anc/photonics/rays.html.

Figure 1: Example problems using Java Applet for Lasers and Photonics 


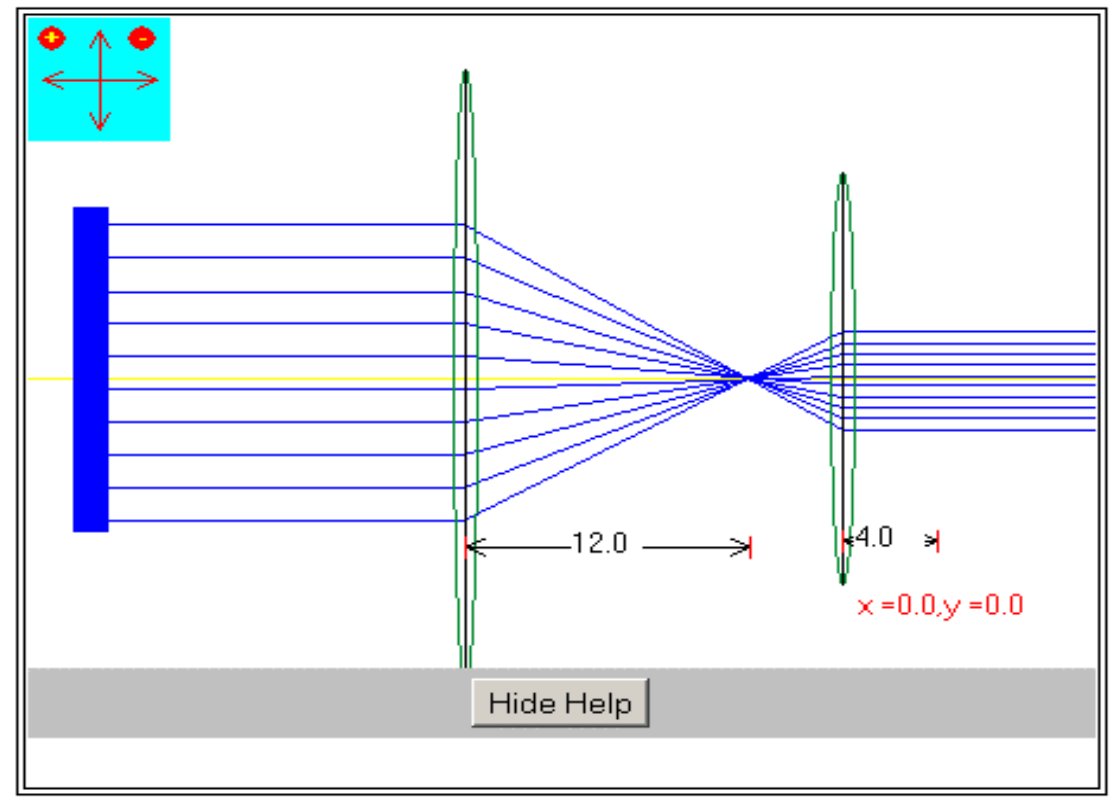

Figure 2: Example solution to Problem 1 from Figure 1

A few example design applets commonly used during the course time are shown in Figures 3 and 4. The design tool shown in Figure 3 can be used to implement any simple optical system. In this applet the user can move, resize, add or drop mirrors, lenses, dielectrics and light sources dynamically to design any system. Moreover, a form based version of the same design tool is shown in Figure 4, wherein the user enters all the values in the blank spaces and the applet constructs the system according to the parameters.

\section{Optical System Design: Version 2.0}

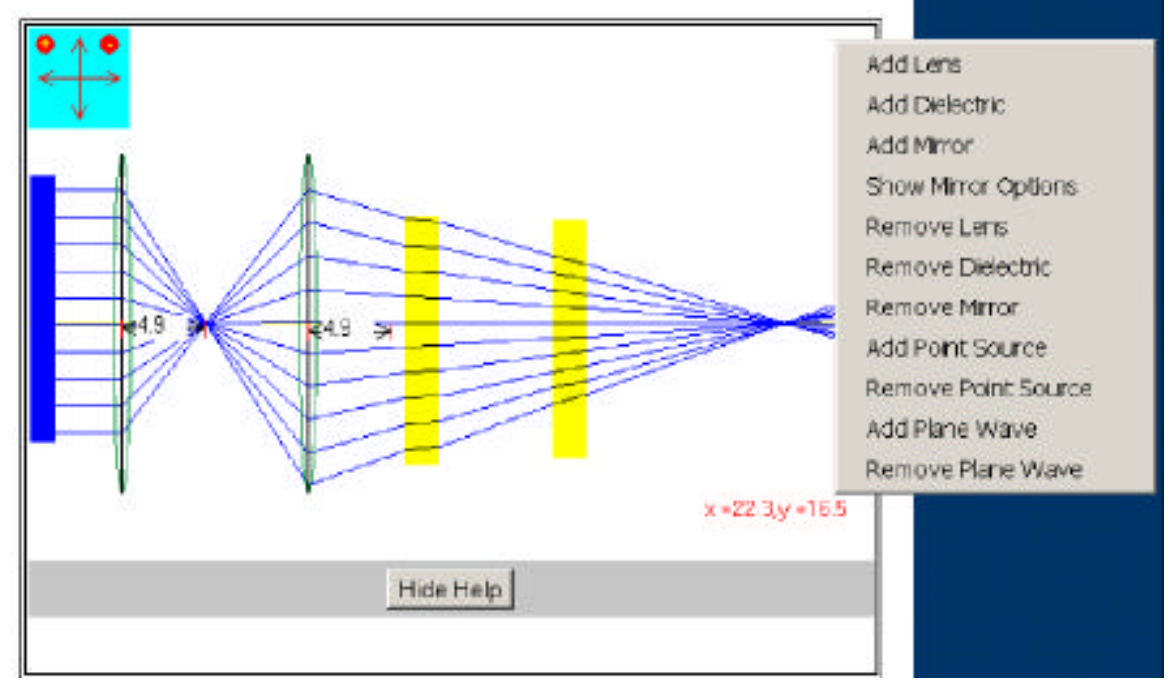

"Proceedings of the 2002 American Society for Engineering Education Annual Conference \& Exposition Copyright (C2002, American Society for Engineering Education” 
Figure 3: Optical Design System Applet

Interactive Optical Design

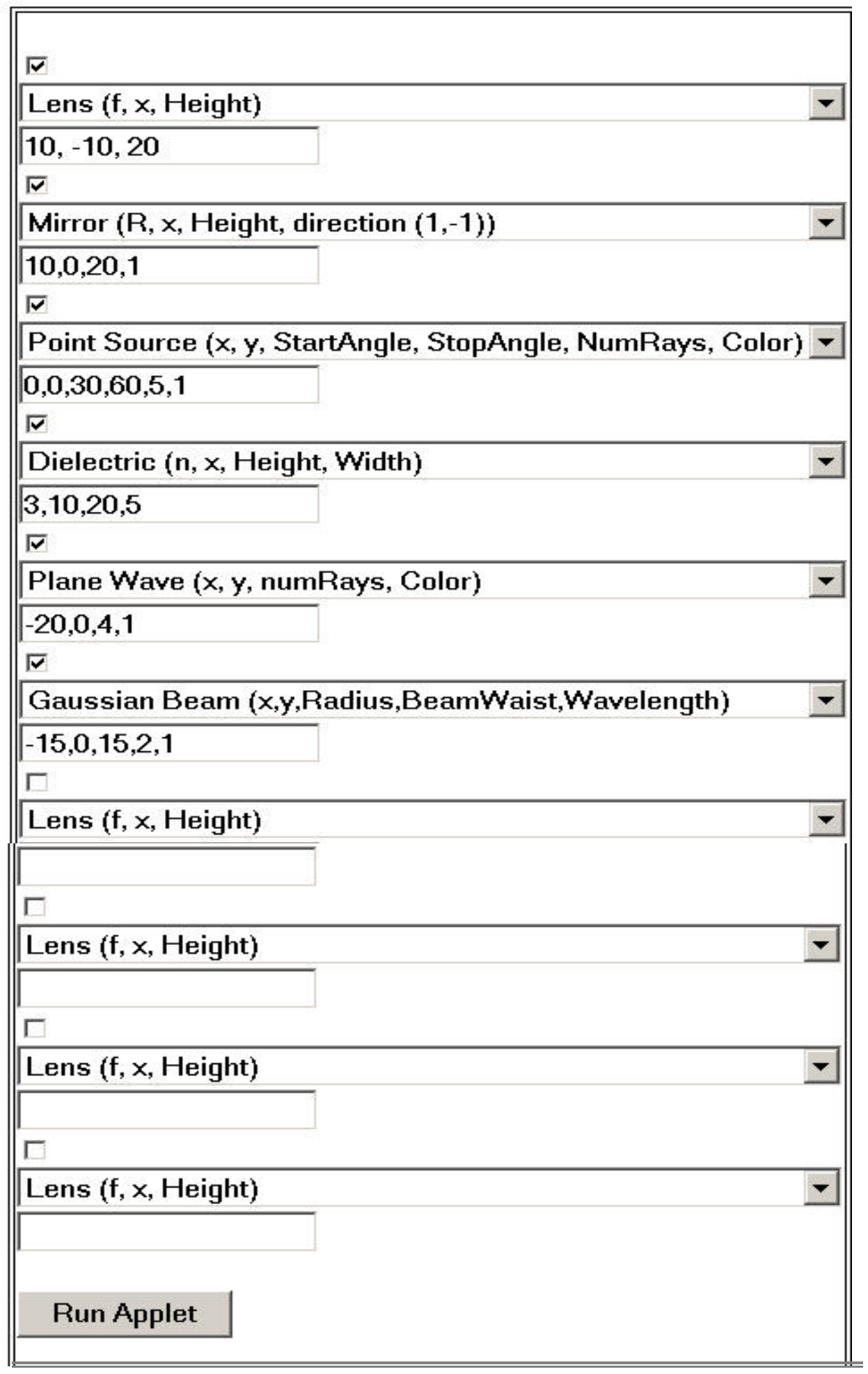

Figure 4: Form-Based Optical Design System Applet

"Proceedings of the 2002 American Society for Engineering Education Annual Conference \& Exposition Copyright (C2002, American Society for Engineering Education” 
For graduate students the main emphasis is on the design of complex systems such as CD-ROM's, scanners and displays so that they are prepared for the engineering-level positions in this exciting area. In the initial course, "Optical Communications (EE566)" the students do a research project and submit a report as their final course project. The projects mainly deal with the design criteria and requirements for the current generation of optical systems used in communications, networking, devices, and various other applications. During the course the students learn the design aspects and principles of various photonic devices like optical modulators and resonator cavities that are used in optoelectronic equipment. A few example questions based on modulators are given in Figure 5. Example applets for an acousto-optic modulator and an electro-optic modulator are shown in Figures 6 and 7 respectively. In the applet shown in Figure 6, the user can vary such parameters as the acousto-optic material, the frequency of the sound wave, and the wavelength and incident angle of the light. The resulting output that the user observes is the angle of the diffracted beam and also the ratio of the intensities of the diffracted and the incident beams (i.e., the diffraction efficiency). In the applet shown in Figure 7 , the user can vary the voltage applied to the crystal, the length of the crystal, the wavelength and the polarization of the input light. The output of the system gives the current state of the polarization of the output light, the relative phase and the ratio of the intensities of the output and the input beams (i.e., the modulation efficiency).

"Use the Acousto-Optic modulator applet to do the following problem. Submit a printout of the completed solutions."

\section{Problem:}

Design an acousto-optic modulator to get an output angle for the diffracted beam between $20^{\circ}$ and $21.3^{\circ}$ and at the same time get a diffraction efficiency between $80 \%$ and $90 \%$. Explain how the diffraction efficiency various with the wavelength. Give a list of the materials that can be used to get the above values.

"Use the Electro-Optic modulator applet to do the following problem. Submit a printout of the completed solutions."

\section{Problem:}

Design an electro-optic modulator to get a modulation efficiency between $40 \%$ and $41 \%$. Also explain how the polarization of the output beam changes by increasing the voltage applied to crystal.

Figure 5: Example problems designed for Optical Communications

"Proceedings of the 2002 American Society for Engineering Education Annual Conference \& Exposition Copyright (C2002, American Society for Engineering Education” 


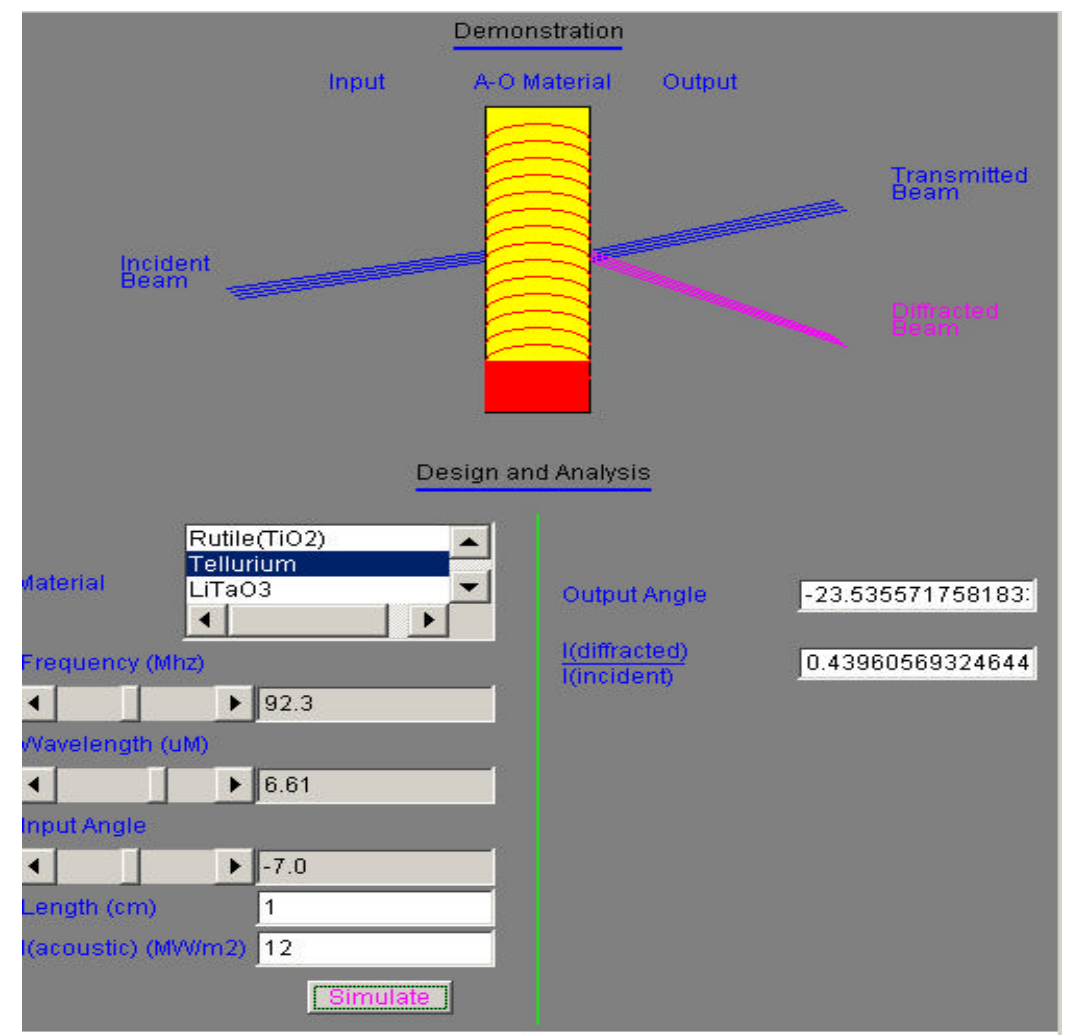

Figure 6: Acousto-Optic Modulator Applet

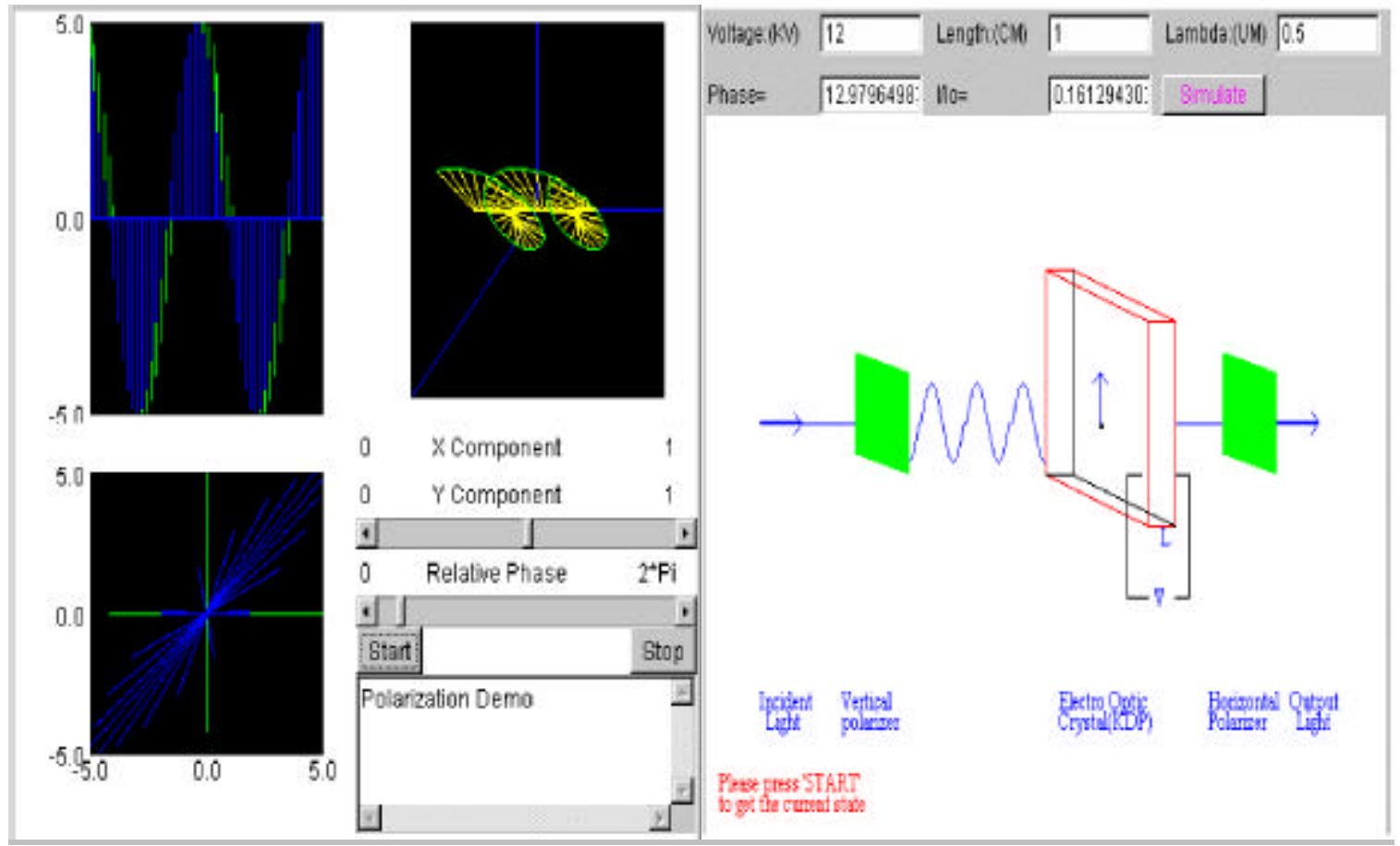

Figure 7: Electro-Optic Modulator Applet

"Proceedings of the 2002 American Society for Engineering Education Annual Conference \& Exposition Copyright (C2002, American Society for Engineering Education” 
In the third course "Consumer Optoelectronics (EE 494 \& 594)" the students are challenged to design a completely new optical system that can replace an existing electronic device as their final course project. This course is for graduate and undergraduate students and is generally quite challenging for the students. In fact, most groups end up designing and rebuilding existing optoelectronic systems. Student projects have included fiber based photoluminescence collection, optical coherence tomography, barcode scanners, optical fiber interconnects and security systems. A few projects have been more innovative, for example the demonstration of a proof of principle device that functioned as a keypad based on the transmission of light through a compressed fiber.

In addition to the required design methodology that is incorporated, these courses require oral and written reports, team efforts, and library research. Thus, the outcomes of these courses that are achieved for graduate and undergraduate students are that they have:

An ability to design various photonic systems to meet the prescribed requirements as well as analyze and interpret the outcome.

An ability to understand and solve various open-ended problems underlying today's high-tech photonic devices.

$>$ An ability to work in teams with members from different backgrounds.

$>$ An ability to communicate freely by participating in various presentations for their respective projects.

> An ability to use the World Wide Web and multimedia technologies to broaden the understanding and knowledge of the principles and fundamentals of photonic devices.

More interest to pursue a career in the area of photonics.

\section{Conclusion and Future Developments}

This new learning environment has been developed during the past 3 years to aid in the teaching of photonics and laser courses at the University at Buffalo. The response and feedback to these applets has been very supportive both from the students and also from the faculty from other universities across the country. In the near future, we are planning to develop a comprehensive design tool for optical networking and its related applications.

Employing this learning environment built on World Wide Web-based educational tools and multimedia technologies may not be a panacea for all the problems faced by the students in understanding, and also by the teachers in teaching, the principles of photonics. However, we feel that these tools contribute to a better understanding of a complex field that has its roots spread over wide range of areas like physics, material science, mathematics and integrated electronics. We believe that the environment will definitely provide the best opportunities for the students to understand and experiment with the fundamental principles underlying today's high-tech photonic devices. Finally,

"Proceedings of the 2002 American Society for Engineering Education Annual Conference \& Exposition Copyright (C2002, American Society for Engineering Education” 
as stated earlier, we will continue to try to incorporate all the developed Java applets and the web-based courseware materials into a system-level top-down approach so that the students can visualize completely the construction and the operation of most commonly encountered photonic and optoelectronic devices.

\section{Acknowledgment}

The authors would like to acknowledge the financial support of the National Science Foundation, Award \#9950794 and Cartwright's National Science Foundation CAREER Award \#9733720.

\section{References}

1. Alexander N. Cartwright, "Design and Collaborative-learning in Lasers and Photonic Courses", American Society for Engineering Education Annual Conference, Session 2632, 1999.

2. David R. Wallace, Philip Mutooni, "A Comparative Evaluation of World Wide Web-based and Classroom Teaching", Journal of Engineering Education, vol. 86, no. 3,1997 , pp. 211-219.

3. URL: http://www.abet.org/images/Criteria/2002-03EACCriteria.pdf

4. Pratibha Gopalam, Alexander N. Cartwright and Bina Ramamurthy, "Java Enabled Opto-Electronic Learning Tools and A Supporting Framework", American Society for Engineering Education Annual Conference, Session 3532, 2001.

5. Alexander N. Cartwright, Pratibha Gopalam, N. Liu, Z. Yuan, T. Tang and Chu R. Wie, "Context Based Educational Java Applets Using Consumer Products", American Society for Engineering Education Annual Conference, Session 2632, 2000.

6. URL: http://www-ee.eng.buffalo.edu/ anc/photonics/index.html

\section{ALEXANDER N. CARTWRIGHT}

Alexander N. Cartwright received his Ph.D. from the University of Iowa in 1995 and is an Associate Professor in Electrical Engineering Department at the University at Buffalo. In 1998, he received a NSF CAREER Award that supports his research on GaN based optoelectronic devices and his educational activities. In 2000, he was awarded a Department of Defense Young Investigator Award for research in piezoelectricity in III$\mathrm{N}$ materials.

\section{PRATIBHA GOPALAM}

Pratibha Gopalam received her M.S. in Electrical Engineering from the University at Buffalo in 2001. She worked as a Software Engineer for Hewlett Packard hdia Software Operations and Asea Brown Boveri Pvt. Ltd. before joining the University at Buffalo. She is now working with Philips (India) Pvt. Ltd.

"Proceedings of the 2002 American Society for Engineering Education Annual Conference \& Exposition Copyright (C2002, American Society for Engineering Education” 


\section{VAMSY P. CHODAVRAPU}

Vamsy P. Chodavarapu is a graduate student in Electrical Engineering Department at the University at Buffalo. He received his undergraduate degree in Electronics and Instrumentation Engineering from Osmania University, India, in 2001. He is now researching the framework development for the creation of design and learning tools in photonics using Java ${ }^{\mathrm{TM}}$ Applets.

\section{BILL KIRKEY}

Bill Kirkey is working for his Ph.D. in Electrical Engineering Department at the University at Buffalo. He received his B.S. in Electrical Engineering from the University at Buffalo in 2000. His research is currently focused on Biophotonics. 